\title{
The Evaluation of Discrete Barrier Options in a Path Integral Framework
}

\author{
Carl Chiarella ${ }^{1}$, Nadima El-Hassan ${ }^{1}$, and Adam Kucera ${ }^{2}$ \\ 1 School of Finance and Economics, University of Technology, Sydney, PO Box \\ 123, Broadway NSW 2007 Australia carl.chiarella@uts.edu.au, \\ nadima.el-hassan@uts.edu. au \\ 2 Integral Energy, Australia adam.kucera@integral.com.au
}

Summary. The pricing of discretely monitored barrier options is a difficult problem. In general, there is no known closed form solution for pricing such options. A path integral approach to the evaluation of barrier options is developed. This leads to a backward recursion functional equation linking the pricing functions at successive barrier points. This functional equation is solved by expanding the pricing functions in Fourier-Hermite series. The backward recursion functional equation then becomes the backward recurrence relation for the coefficients in the FourierHermite expansion of the pricing functions. A very efficient and accurate method for generating the pricing function at any barrier point is thus obtained.

A number of numerical experiments with the method in order to gain some understanding of the nature of convergence. Results are performed for various volatility values and different numbers of basis functions in the Fourier-Hermite expansion are presented. Comparisons are given between pricing of discrete barrier option in the path integral framework and by use of finite difference methods.

Key words: Discrete Barrier Options; Path Integral; Fourier-Hermite Polynomials; Backward Recursion Method

Acknowledgement. The authors thank the Editors for the invitation to contribute to this special volume dedicated to the 60th birthday of their good friend and colleague Manfred Gilli. The authors would also like to thank the referees for comments on the first draft which led to an improved final version. 


\section{Introduction}

Barrier options are derivative securities with values contingent on the relationship between the value of the underlying asset and one or more barrier levels. In this paper we consider the pricing of barrier options which are monitored at particular points over the life of the contract, also known as discrete barrier options. These types of exotic options have become a prominent feature of modern financial markets with many variations heavily traded in the foreign exchange, equity and fixed income markets. The emergence of such securities has also provided challenging research problems in the area of efficient pricing and hedging of such securities. Most pricing models (Merton (1973), Rubinstein and Reiner (1991), Heynen and Kat (1996)) consider barrier options whose barrier level is continuously monitored at every instant in time over the life of the option, allowing the derivation of closed form solutions. However most traded barrier options are monitored discretely, rather than on a continuous basis. The application of the solutions derived assuming continuous monitoring to the pricing of discretely monitored barrier options results in substantial pricing errors (Chance (1994), Kat (1995) and Levy and Mantion (1997)). Hence the need for a method to accurately and efficiently evaluate discretely monitored barrier options. Traditional lattice and Monte Carlo methods have difficulties in incorporating discrete monitoring principally because of the misalignment of the monitoring points.

Several papers have appeared in the literature proposing various methods to handle discrete monitoring. Variations to the traditional binomial and trinomial methods were proposed by Figlewski and Gao (1997), Tian (1996) and Boyle and Tian (1997). These include Broadie, Glasserman and Kou (1997a) who propose a method based on the price of a continuous barrier options with a continuity correction for discrete monitoring. Broadie, Glasserman and Kou (1997b) incorporate the correction term in developing a lattice method for determining accurate prices of discrete and continuous path-dependent options. As far as the pricing of discretely monitored barrier options is concerned Wei (1998) proposes an interpolation method whereas Sullivan (2000) proposes a method that reduces the discrete time multidimensional integration required to a sequential numerical integration.

In this paper we examine a potentially powerful alternative to existing pricing methods. Using knowledge of the conditional transition density function we repeatedly apply the Chapman-Kolmogorov equation to relate the pricing function at successive monitoring points. We then expand the pricing function in a Fourier-Hermite series in terms of the price of the underlying asset. We derive recurrence relations involving orthogonal polynomials under a given measure. The proposed method works well for various discrete barrier structures including, single and double barriers, constant and time varying barrier levels and for a number of payoff structures such as vanillas, digital and powers. The method can be made arbitrarily accurate by taking a sufficient number of terms in the expansion. 
The techniques used extend the work of Chiarella, El-Hassan and Kucera (1999) where Fourier-Hermite series expansions were applied to the valuation of European and American options. The novel aspect of our contribution is the expansion of the derivative security price at each time step in a FourierHermite series expansion so that it is obtained as a continuous and differentiable function of the price of the underlying asset. The actual implementation of our method then becomes a question of determining the coefficients of the Fourier-Hermite series expansion at each time step. Using the orthogonalisation condition, it turns out that these can be generated recursively by working backwards from one time step to the next, by use of the recurrence relations which generate the Hermite polynomials. The implementation of these recurrence relations is in fact very efficient. Here we apply the method to pricing discretely monitored barrier options. Since we obtain a continuous and differentiable representation of the price, the hedge ratios delta and gamma can be obtained to a high level of accuracy and also very cheaply in terms of calculation time.

Our approach may be regarded as one way to implement the path integral techniques in option pricing problems. In recent years it has become appreciated that the path integral technique of statistical physics can be applied to derivative security valuation. We refer in particular to Linetsky (1997) who provides an overview of the path integral concept and its application to financial problems. The wide application of path integral techniques to financial modelling and in particular to the pricing and hedging of options, including path-dependent and exotic options, was first studied by Dash (1988). Dash's contribution to the area was largely in the formulation of many derivative security pricing problems in the path integral framework, including standard equity options, exotic options, path dependent options and the pricing of bond options under a number of popular term structure models. The framework provides an intuitive description of the value of derivative securities using relatively simple mathematics.

However, the application and implementation of solution techniques of path integrals to finance problems has been limited, with the cited authors focusing on a general framework and pointing to the potential for the application of these techniques to financial pricing problems. Path integrals can be evaluated in a number of ways including analytic approximations by means of moment expansions in a perturbation series, deterministic discretisation schemes of the path integral and Monte-Carlo simulation (Makici (1995)) methods. The method chosen will in general depend on the problem at hand and the stability of the solution technique used. We also refer to the contribution of Eydeland (1994) who provides a computational algorithm based on a Toeplitz matrix structure and fast Fourier transforms for evaluating financial securities in a path integral framework. This technique was successfully applied by Chiarella and El-Hassan (1997) to evaluate European and American bond options in an HJM framework. 
The organisation of the paper is as follows: In section 2, we define the general barrier structure and outline the backward recursion procedure. In section 3 , we show how the path integral formulation for the value of a discretely monitored barrier defined in section 2 can be represented as an expansion of the value function in a series of orthogonal polynomials and reduced to a backward recursion procedure. In section 4 , we report some numerical results and in section 5 we make some concluding remarks.

\section{The General Barrier Structure}

We denote the underlying asset price by $S$ and assume that under the riskneutral measure it follows a geometric Brownian motion given by

$$
d S=(r-q) S d t+\sigma S d W
$$

where $\sigma$ is the volatility, $r$ is the risk-free rate of interest, $q$ is the continuous dividend yield on the underlying asset and $W(t)$ is a standard Wiener process. By allowing for a continuous dividend yield $q$ our framework may be applied to barrier options on indices, or to options on foreign exchange by setting $q=r_{f}$ where $r_{f}$ is the risk free rate of interest in the foreign economy.

The implementation of the path integral method described below follows the framework laid out in Chiarella et al. (1999), which requires expansion of pricing functions in terms of Hermite polynomials. These are defined on an infinite interval, for this reason we need to transform the asset price to a variable defined on an infinite interval. This is most conveniently done by introducing the change of variable

$$
\xi=\frac{1}{\sigma} \ln (S) .
$$

A straight forward application of Ito's lemma reveals that $\xi$ satisfies the stochastic differential equation

$$
d \xi=\frac{1}{\sigma}\left((r-q)-\frac{1}{2} \sigma^{2}\right) d t+d W(t) .
$$

We recall that (2) implies that the transition probability density for $\xi$ between two times $t^{\prime}, t\left(t^{\prime}<t\right)$, denoted $\pi\left(\xi_{t}, t \mid \xi_{t^{\prime}}, t^{\prime}\right)$ is normally distributed and is in fact given by

$$
\pi\left(\xi_{t}, t \mid \xi_{t^{\prime}}, t^{\prime}\right)=\frac{1}{\sqrt{2 \pi\left(t-t^{\prime}\right)}} \exp \left[\frac{-\left[\xi_{t}-\sqrt{2\left(t-t^{\prime}\right)} \mu\left(\xi_{t^{\prime}}, t-t^{\prime}\right)\right]^{2}}{2\left(t-t^{\prime}\right)}\right],
$$

where it is convenient to define 


$$
\mu(\xi, t)=\frac{1}{\sqrt{2 t}}\left(\xi+\frac{1}{\sigma}\left(r-q-\frac{1}{2} \sigma^{2}\right) t\right) .
$$

We divide the time interval from initial time to option maturity into $K$ subintervals $\left(t^{k-1}, t^{k}\right),(k=0,1, \cdots, K)$. The spacings between the barrier observation points, $t^{k}$, need not be constant. We set $\Delta t^{k}=t^{k}-t^{k-1}$ for $k=K, \cdots, 1$ with the implied notation that $t^{0}=0$ and $t^{K}=T$ so that $T=\sum_{k=1}^{K} \Delta t^{k}$.

We allow for barrier levels that can be time dependent. Thus at each time $t^{k}$ there will be an upper barrier level, $b_{u}^{k}$, and a lower barrier level $b_{l}^{k}$, for $k=K-1, \cdots 1$ Here we note that we do not include barriers at expiry since they are part of the pay-off definition. With this notation we may handle the case of no lower barrier by setting $b_{l}^{k}=0$, and the case of no upper barrier by letting $b_{u}^{k} \rightarrow \infty$. Figure 1 illustrates in the $S, t$ plane a typical discretisation with a variety of possible barriers at the discretisation points.

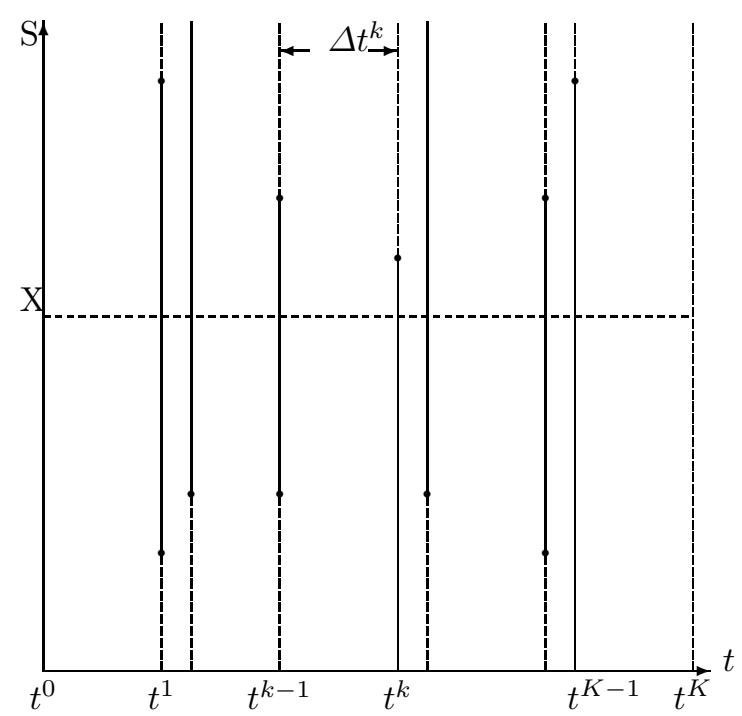

Fig. 1. The Discretisation Scheme 


\section{THE BARRIER OPTION AS A functional recurrence relation EQUATION}

Under the risk neutral measure the price of a derivative security at any point in time is the discounted expected payoff at the next point at which it may be exercised. The transition probability density function (3) is the one that is required to calculate the expected payoff. We use $F^{k}\left(\xi^{k}\right)$ to denote the value function of the barrier option at monitoring point $t^{k}$ as a function of $\xi^{k}$, the volatility scaled $\log$ price. Then the discounted expected value relation between the value functions at two successive monitoring points $t^{k-1}, t^{k}$ is given by

$$
F^{k-1}\left(\xi^{k-1}\right)=e^{-r \Delta t^{k}} \int_{\ln \left(b_{l}^{k}\right) / \sigma}^{\ln \left(b_{n}^{k}\right) / \sigma} \pi\left(\xi^{k}, t^{k} \mid \xi^{k-1}, t^{k-1}\right) F^{k}\left(\xi^{k}\right) d \xi^{k} .
$$

Substituting equation (3) and making a change of integration variable we obtain

$$
F^{k-1}\left(\xi^{k-1}\right)=\frac{e^{-r \Delta t^{k}}}{\sqrt{\pi}} \int_{z_{l}^{k}}^{z_{u}^{k}} e^{-\left(\xi^{k}-\mu\left(\xi^{k-1}, \Delta t^{k}\right)\right)^{2}} F^{k}\left(\sqrt{2 \Delta t^{k}} \xi^{k}\right) d \xi^{k},
$$

where, due to the the scaling factor $\sqrt{2 \Delta t^{k}}$ in $F^{k}$, the limits of integration $z_{l}^{k}$ and $z_{u}^{k}$ are given by

$$
z_{l}^{k}=\frac{\ln \left(b_{l}^{k}\right)}{\sigma \sqrt{2 \Delta t^{k}}} \quad \text { and } \quad z_{u}^{k}=\frac{\ln \left(b_{u}^{k}\right)}{\sigma \sqrt{2 \Delta t^{k}}},
$$

for $k=K-1 \cdots, 1$. Thus the notation implicitly carries the time dependance of the problem. With the above notation, equation (6) can be written as

$$
F^{k-1}\left(\xi^{k-1}\right)=\frac{e^{-r \Delta t^{k}}}{\sqrt{\pi}} \int_{z_{l}^{k}}^{z_{u}^{k}} e^{-\left(\xi^{k}-\mu\left(\xi^{k-1}, \Delta t^{k}\right)\right)^{2}} F^{k}\left(\sqrt{2 \Delta t^{k}} \xi^{k}\right) d \xi^{k},
$$

Equation 8 is the functional recurrence equation that we need to solve. We note that in stepping back the range of integration, from $t=t^{k}$ to $t=t^{k-1}$ (i.e. propagating back the solution) is given by $0 \leq \xi^{k} \leq \infty$ for a call option and for a put option by $-\infty<\xi^{k} \leq 0$. In this way, the max function is handled by the integration limits. For ease of clarity and notation, when performing the path integrations across all the time intervals, we will denote $\xi^{k}$ as $x, \xi^{k-1}$ as $\xi, z_{l}^{k}$ as $z_{l}, z_{u}^{k}$ as $z_{u}, \Delta t^{k}$ as $\Delta t$.

With the above notation, equation (1.7) can be written as

$$
F^{k-1}(\xi)=e^{-r \Delta t} \frac{1}{\sqrt{\pi}} \int_{z_{l}}^{z_{u}} e^{-(x-\mu(\xi, \Delta t))^{2}} F^{k}(\sqrt{2 \Delta t} x) d x, \quad k=K, \ldots, 1 .
$$


Figure 2 illustrates the region of integration and the concept of the backward propogation of the price function. The task at hand involves successive iteration of equation (9) from the given pay-off function, $F^{k}\left(\xi^{k}\right)$ through the barrier points back to $t=0$. On completing the iterations, it is a simple matter to invert the log transformation and return to the price variable $\mathrm{S}$ and evaluate $F^{0}$ at the required spot value.

Because of our geometric Brownian motion assumption for the underlying asset price the option pricing function is homogeneous in $\frac{S}{X}$. Hence for a call option we need only consider a pay-off function of the form $\max (S-1,0)$ and a put option a pay-off function $\max (1-S, 0)$. Under the log transformation the pay-off for a call option becomes $\max \left[0, e^{\sigma^{k}} \xi^{k}-1\right]$. Similarly the pay-off function for a put option is $\max \left[0,1-e^{\sigma^{k}} \xi^{k}-1\right]$. We use $F^{K}\left(\xi^{K}\right)$ to denote the payoff function at the final time $t^{K}$.

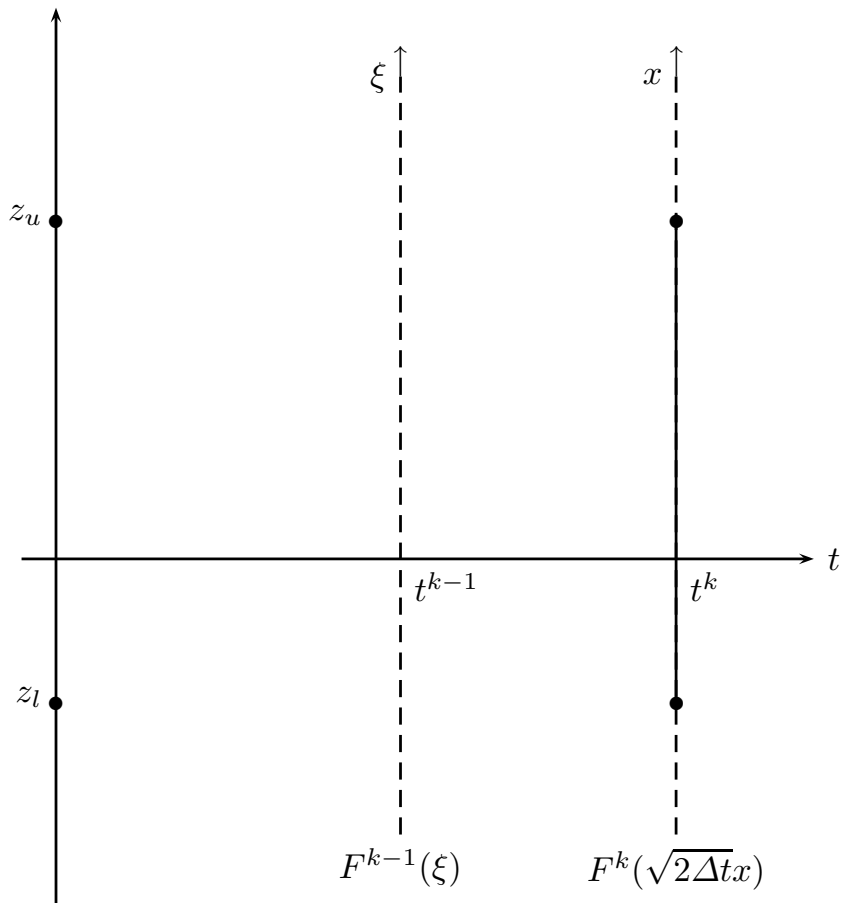

Fig. 2. Propagating the Price Function Back from $t^{k}$ to $t^{k-1}$ 


\section{The Fourier-Hermite series Expansion}

The basic problem in implementing 9 is to obtain a convenient way to evaluate the integral on the right-hand side and to then build up successively the value functions $F^{k}(\xi)(k=K-1, \cdots, 1)$. In this paper we solve this problem by expanding both $F^{k}(x)$ and $F^{k-1}(\xi)$ in Fourier-Hermite series . Thus we set

$$
\left.\begin{array}{c}
F^{k}(x) \approx \sum_{n=0}^{N} \alpha_{n}^{k} H_{n}(x), \\
F^{k-1}(\xi) \approx \sum_{m=0}^{N} \alpha_{m}^{k-1} H_{m}(\xi),
\end{array}\right\}
$$

for $\mathrm{N}$ sufficiently large to ensure convergence of the series. By substituting the Fourier-Hermite expansions (10) into the backward recurrence 9 and making use of the orthogonality property of Hermite polynomials, it is possible to obtain a backward recurrence relation for the coefficients in the expansions (10). In this way, we are able to construct the value functions $F^{k}(\xi)$. We state this key result in proposition I, but before stating this proposition it is useful to introduce some special notation for quantities which occur frequently in the calculations below.

\section{Notation $^{3}$}

First introduce the functions

$$
L_{m}(x)=\frac{H_{m}(x)}{2^{m} m ! v^{m}}
$$

which are easily shown to satisfy the recurrence equation

$$
L_{m}(x)=\frac{x L_{m-1}(x)}{m v}-\frac{L_{m-2}(x)}{2(m-1) v^{2}},
$$

with $L_{0}(x)=1$ and $L_{1}(x)=x / v$.

Next we set (recall $\left.\phi(x):=e^{-x^{2} / 2} / \sqrt{2 \pi}\right)$

$$
R_{m, n}(x)=\sqrt{2} L_{m}(x) H_{n}(v x+\beta) \phi(\sqrt{2} x),
$$

from which we define

$$
Q_{m, n}(x, y)=R_{m, n}(x)-R_{m, n}(y) .
$$

Finally we define

$$
P(x, y)=\Phi(\sqrt{2} x)-\Phi(\sqrt{2} y)
$$

${ }^{3}$ See Appendix $\mathrm{H}$ for a summary of the notation. 
where

$$
\Phi(x)=\frac{1}{\sqrt{2 \pi}} \int_{-\infty}^{x} e^{-\frac{u^{2}}{2}} d u,
$$

is the cumulative normal density function.

\section{Proposition I}

The known coefficients $\alpha_{n}^{k}$ and the to be calculated coefficients $\alpha_{m}^{k-1}$ at the time $t^{k-1}$ are connected by the recurrence relation

$$
\alpha_{m}^{k-1}=e^{-r \Delta t} \sum_{n=0}^{N} a_{m, n}^{k} \alpha_{n}^{k}, \quad \text { for } \quad k=K, \cdots, 1
$$

or in matrix notation:

$$
\boldsymbol{\alpha}^{k-1}=e^{-r \Delta t} A^{k} \boldsymbol{\alpha}^{k}, \quad \text { for } \quad k=K, \cdots, 1
$$

where

$$
A^{k}=\left[a_{m, n}^{k}\right] \quad \text { for } \quad m=0,1, \cdots, N \quad \text { and } \quad n=0,1, \cdots, N
$$

The coefficients $a_{m, n}^{k}$ are generated by the equations outlined in the proof below.

Proof. Substituting the two series expansions (10) into the functional equation 9 , using the orthogonality properties of the Hermite polynomials we find after some algebraic manipulations that

$$
\alpha_{m}^{k-1}=e^{-r \Delta t} \sum_{n=0}^{N} \frac{n}{2^{m} m !} \frac{\alpha_{n}^{k}}{\sqrt{\pi}} \int_{z_{l}}^{z_{u}} H_{n}(\sqrt{2 \Delta t} x) I_{m}(x) d x,
$$

where

$$
I_{m}(x)=\frac{\sqrt{2 \Delta t}}{v^{m+1}} H_{m}\left(\frac{\sqrt{2 \Delta t} x-\beta}{v}\right) \exp \left[-\left(\frac{\sqrt{2 \Delta t} x-\beta}{v}\right)^{2}\right],
$$

with

$$
v=\sqrt{1+2 \Delta t} \quad \text { and } \quad \beta=\frac{1}{\sigma}\left(r-q-\frac{1}{2} \sigma^{2}\right) \Delta t .
$$

Now, introducing the transformation

$$
z=\frac{\sqrt{2 \Delta t} x-\beta}{v}
$$

equation (19) can be written as

$$
\alpha_{m}^{k-1}=e^{-r \Delta t} \sum_{n=0}^{N} a_{m, n}^{k} \alpha_{n}^{k}
$$


where the elements $a_{m, n}^{k}$ are given by

$$
a_{m, n}^{k}=\frac{1}{2^{m} m ! v^{m}} \frac{1}{\sqrt{\pi}} \int_{x_{l}}^{x_{u}} e^{-x^{2}} H_{m}(x) H_{n}(v x+\beta) d x .
$$

The new limits of integration $x_{l}$ and $x_{u}$ are given by

$$
x_{l}=\frac{\sqrt{2 \Delta t} z_{l}-\beta}{v} \quad \text { and } \quad x_{u}=\frac{\sqrt{2 \Delta t} z_{u}-\beta}{v} .
$$

We note that at final time $t^{K}$ for a call, the integration limits become

$$
x_{l}=0 \quad \text { and } \quad x_{u} \rightarrow \infty,
$$

whilst for a put, when $k=K$,

$$
x_{l} \rightarrow-\infty \quad \text { and } \quad x_{u}=0 .
$$

At final time $t^{K}$, it is convenient to define $w$ such that $w=1(-1)$ applies to a Call(Put).

Thus taking into consideration the foregoing limits the first four elements are given by

$$
\begin{aligned}
& a_{0,0}^{K}=\frac{w}{\sqrt{\pi}} \int_{0}^{w \infty} e^{-x^{2}} d x=\frac{1}{2} \\
& a_{0,1}^{K}=\frac{w}{\sqrt{\pi}} \int_{0}^{w \infty} e^{-x^{2}} 2(v x+\beta) d x=\frac{w}{2 \sqrt{\pi}}+\beta \\
& a_{1,0}^{K}=\frac{w}{\sqrt{\pi}} \int_{0}^{w \infty} e^{-x^{2}} 2 x d x=\frac{w}{2 v \sqrt{\pi}} \\
& a_{1,1}^{K}=\frac{w}{\sqrt{\pi}} \int_{0}^{w \infty} e^{-x^{2}}(2 x) 2(v x+\beta) d x=\frac{1}{2}+\frac{w \beta}{v \sqrt{\pi}} .
\end{aligned}
$$

For $m=2,3, \cdots, N$ with $n=0$

$$
a_{m, 0}^{K}=\frac{w}{2^{m} m ! v^{m} \sqrt{\pi}} \int_{0}^{w \infty} e^{-x^{2}} H_{m}(x) d x=\frac{w}{2 m v \sqrt{\pi}} L_{m-1}(0) .
$$

For $n=2,3, \cdots, N$ with $m=0$

$$
a_{0, n}^{K}=\frac{w}{\sqrt{\pi}} \int_{0}^{w \infty} e^{-x^{2}} H_{n}(v x+\beta) d x,
$$

which upon use of the recurrence equation for Hermite polynomials yields the recurrence equation

$$
a_{0, n}^{K}=\frac{w v}{\sqrt{\pi}} H_{n-1}(\beta)+2 \beta a_{0, n-1}^{K}+2\left(v^{2}-1\right)(n-1) a_{0, n-2} .
$$


For $m=2,3, \cdots, N$ and $n=2,3, \cdots, N$ application of the recurrence equation for Hermite polynomials yields

$$
a_{m, n}^{K}=\frac{w \sqrt{2}}{2 m v \sqrt{\pi}} L_{m-1}(0) H_{n}(\beta)+\frac{n}{m} a_{n-1, m-1}^{K} .
$$

Next we consider generation of the coefficients $a_{m, n}^{k}$ at any general time step $k$.

Using the definitions of $P$ and $Q$ from (14) and (15), then the first four elements are given by

$a_{0,0}^{k}=\frac{1}{\sqrt{\pi}} \int_{x_{l}}^{x_{u}} e^{-x^{2}} d x=P\left(x_{u}, x_{l}\right), \quad($ see appendix $\mathrm{A})$

$a_{1,0}^{k}=\frac{1}{2 v \sqrt{\pi}} \int_{x_{l}}^{x_{u}} e^{-x^{2}} 2 x d x=\frac{1}{2 v} Q_{0,0}\left(x_{l}, x_{u}\right), \quad($ see appendix B $)$

$a_{0,1}^{k}=\frac{1}{\sqrt{\pi}} \int_{x_{l}}^{x_{u}} e^{-x^{2}} 2(v x+\beta) d x=v Q_{0,0}\left(x_{l}, x_{u}\right)+2 \beta P\left(x_{u}, x_{l}\right), \quad$ (see appendix C)

$a_{1,1}^{k}=\frac{1}{2 v \sqrt{\pi}} \int_{x_{l}}^{x_{u}} e^{-x^{2}}(2 x) 2(v x+\beta) d x=\frac{1}{2 v} Q_{0,1}\left(x_{l}, x_{u}\right)+P\left(x_{u}, x_{l}\right) . \quad$ ( see appendix D)

To alleviate the notation we shall henceforth set

$$
P=P\left(x_{u}, x_{l}\right) \quad \text { and } \quad Q_{m, n}=Q_{m, n}\left(x_{l}, x_{u}\right) .
$$

For $m=2,3, \cdots, N$ with $n=0$ (see appendix E)

$$
a_{m, 0}^{k}=\frac{1}{2 m v} Q_{m-1,0} .
$$

For $n=2,3, \cdots, N$ with $m=0$ (see appendix $\mathrm{F}$ )

$$
a_{0, n}^{k}=v Q_{0, n-1}+2 \beta a_{0, n-1}^{k}+2\left(v^{2}-1\right)(n-1) a_{0, n-2}^{k} .
$$

For $m=2,3, \cdots, N$ and $n=2,3, \cdots, N$ (see appendix G)

$$
a_{m, n}^{k}=\frac{1}{2 m v} Q_{m-1, n}+\frac{n}{m} a_{m-1, n-1}^{k} .
$$

Alternatively, if we introduce a new function

$$
Z_{m, n}=\frac{1}{2(m+1) v} Q_{m, n}\left(x_{l}, x_{u}\right),
$$

then the first four elements are given by

$$
\begin{aligned}
& a_{0,0}^{k}=P \quad a_{0,1}^{k}=2 v^{2} Z_{0,0}+2 \beta P, \\
& a_{1,0}^{k}=Z_{0,0} \quad a_{1,1}^{k}=Z_{0,1}+P .
\end{aligned}
$$


For $m=2,3, \cdots, N$ and $n=0$

$$
a_{m, 0}^{k}=Z_{m-1,0}
$$

For $n=2,3, \cdots, N$ and $m=0$

$$
a_{0, n}^{k}=2 v^{2} Z_{0, n-1}+2 \beta a_{0, n-1}^{k}+2\left(v^{2}-1\right)(n-1) a_{0, n-2}^{k} .
$$

For $m=2,3, \cdots, N$ and $n=2,3, \cdots, N$

$$
a_{m, n}^{k}=Z_{m-1, n}+\frac{n}{m} a_{m-1, n-1}^{k} .
$$

All of the elements of the matrix $A$ of the proposition are now defined.

Proposition I has specified the coefficients of the matrix $A$ which determine the backward transition of the $\boldsymbol{\alpha}^{k}$ coefficients from final time back to initial time. The quantity that we need to initialise the entire backward propogation process is $\boldsymbol{\alpha}^{K}$, the set of $\alpha$ coefficients at final time. These are determined by the payoff function at final time. In fact they are computed by expanding the payoff function itself in a Fourier-Hermite series .

Recalling the payoff functions for calls and puts (see Figure 2) and the notation $w=1(-1)$ to indicate call (put) the backward recursion from final payoff at $t^{k}$ to the time step $t^{K-1}$ may be written

$$
F^{K-1}(\xi)=\frac{e^{-r \Delta t}}{\sqrt{\pi}} \int_{z_{l}}^{z_{u}} e^{-(x-\mu(\xi, \Delta t))^{2}} w\left(e^{\sigma x}-1\right) d x
$$

In the case of a call $z_{u}=\infty$ and $z_{l}=0$. In the case of a put $z_{u}=0$ and $z_{l}=-\infty$. we note however that the notation employed for the limits of integration in (41) also allows us to cater for the situation when there are discrete barriers at final time. In this case $z_{u}$ and $z_{l}$ would be determined by the barrier points.

Thus in order to calculate the coefficients $\alpha_{n}^{K}$ we first need to expand $e^{\sigma x}$ in a Fourier-Hermite series .

First we note the result that

$$
\frac{1}{\sqrt{\pi}} \int_{-\infty}^{\infty} e^{-x^{2}} H_{n}(x) e^{\sigma x} d x=\sigma^{n} e^{\frac{\sigma^{2}}{4}}
$$

Then forming the Fourier-Hermite series

$$
e^{\sigma x}=\sum_{n=0}^{\infty} \beta_{n} H_{n}(x)
$$

we apply the orthogonality condition to obtain

$$
\beta_{n}=\frac{1}{2^{n} n !} \frac{1}{\sqrt{\pi}} \int_{-\infty}^{\infty} e^{-x^{2}} H_{n}(x) e^{\sigma x} d x
$$


which by use of (41) reduces to

$$
\beta_{n}=\frac{\sigma^{n}}{2^{n} n !} e^{\frac{\sigma^{2}}{4}} .
$$

Therefore, for a call,

$$
\alpha_{n}^{K}=\beta_{n} \quad \text { for } \quad n=1,2, \cdots, N
$$

and $\alpha_{0}^{K}=e^{\sigma^{2}}-1$.

While for a Put,

$$
\alpha_{n}^{K}=-\beta_{n} \quad \text { for } \quad n=1,2, \cdots, N
$$

and $\alpha_{0}^{K}=1-e^{\sigma^{2}}$.

\section{Results}

In this section we present some preliminary results obtained from implementing the path integral framework for pricing discretely monitored barrier options. The method was tested for parameter values similar to those used by Broadie et al. (1997a) and Wei (1998). The method was also tested on barrier puts and calls with digital type payoff functions. To ascertain the accuracy of the method, a Crank-Nicholson was used to generate true prices for the options investigated. ${ }^{4}$ Hence, the Crank-Nicholson scheme was used with very fine discretisations, with the stock price variable taken out to five times the value of the strike (scaled to unity) with 2000 steps per unit. The time variable was discretised to 100 steps per day.

\begin{tabular}{|c||c|c|c|}
\hline & $\begin{array}{c}\text { Path Integral } \\
\mathrm{m}=40\end{array}$ & $\begin{array}{c}\text { Path Integral } \\
\mathrm{m}=100\end{array}$ & $\mathrm{CN}$ \\
\hline$\sigma=0.60$ & 9.4895 & 9.4904 & 9.4905 \\
$\sigma=0.40$ & 7.0388 & 7.0393 & 7.0394 \\
$\sigma=0.20$ & 4.4336 & 4.4342 & 4.4344 \\
\hline
\end{tabular}

TABLE 1. Discretely Monitored Down-and-Out Call Option

Parameters: $S=K=100, T=0.2$ year, $r=0.1$,

$q=0$, barrier $=95$, monitoring frequency $=4$.

\footnotetext{
${ }^{4}$ Our implementation of the Crank-Nicholson scheme for discrete barrier options follows the approach outlined in Tavella (2002).
} 
Table 1 gives results of the path integral method for a set of parameter values which differ in their volatility value. The results are presented using 40 and 100 basis functions and are compete with the Crank-Nicholson (CN) true solution using the high order discretisation mentioned earlier. It is evident that even with 40 basis functions the method is relatively accurate when compared with the true values, with the results approaching the true values when using 100 basis functions. Note that the parameter values used in this test include high volatility as well as the barrier level close to the current asset price which typically present problems in other pricing methods for discretely monitored barrier options.

\begin{tabular}{|ccccc|}
\hline & & & \multicolumn{2}{c|}{ Percentage Pricing Error } \\
\cline { 3 - 5 } $\begin{array}{c}\text { Barrier } \\
\text { Level }\end{array}$ & $\begin{array}{c}\text { Path Integral } \\
\text { Solution }\end{array}$ & $\begin{array}{c}\text { CN } \\
\text { Solution }\end{array}$ & $\begin{array}{c}\text { Path } \\
\text { Integral }\end{array}$ & $\begin{array}{c}\text { BGK } \\
\text { Correction }\end{array}$ \\
\hline \multicolumn{5}{c}{ Monthly Monitoring } \\
\hline 85 & 8.1859 & 8.1861 & $-0.003 \%$ & $-0.011 \%$ \\
90 & 7.8406 & 7.8403 & $0.004 \%$ & $-0.008 \%$ \\
95 & 6.7450 & 6.7463 & $-0.019 \%$ & $0.036 \%$ \\
99.5 & 4.9323 & 4.9338 & $-0.031 \%$ & $-10.926 \%$ \\
99.9 & 4.7460 & 4.7474 & $-0.030 \%$ & $-16.023 \%$ \\
\hline \multicolumn{5}{c}{ Weekly Monitoring } \\
\hline 85 & 8.1248 & 8.1250 & $-0.003 \%$ & $0.006 \%$ \\
90 & 7.5761 & 7.5763 & $-0.003 \%$ & $0.080 \%$ \\
99 & 5.8936 & 5.8946 & $-0.016 \%$ & $-0.870 \%$ \\
99.5 & 3.0078 & 3.0093 & $-0.050 \%$ & $-13.570 \%$ \\
\hline
\end{tabular}

\section{TABLE 2. At-the-money Down and Out Call Options}

Parameters: $S=K=100, T=0.5$ year, $r=0.05$,

$$
q=0, \sigma=0.25,100 \text { basis function }
$$

Table 2, table 3 and table 4 show results of the method for at-the-money, out-of-the-money and in-the-money down-and-out options under two monitoring frequencies - monthly and weekly. Results are presented for various barrier levels including values approaching the current asset values. Also presented are percentage-pricing errors relative to the true prices obtained using the Crank-Nicholson method. These give the percentage error of the option prices 


\begin{tabular}{|c|c|c|c|c|}
\hline \multirow{3}{*}{$\begin{array}{c}\text { Barrier } \\
\text { Level }\end{array}$} & \multirow{3}{*}{$\begin{array}{l}\text { Path Integral } \\
\text { Solution }\end{array}$} & \multirow{3}{*}{$\begin{array}{c}\mathrm{CN} \\
\text { Solution }\end{array}$} & \multicolumn{2}{|c|}{ Percentage Pricing Error } \\
\hline & & & Path & BGK \\
\hline & & & Integral & Correction \\
\hline \multicolumn{5}{|c|}{ Monthly Monitoring } \\
\hline 85 & 10.8029 & 10.8052 & $-0.022 \%$ & $-0.010 \%$ \\
\hline 90 & 9.8831 & 9.8865 & $-0.035 \%$ & $-0.007 \%$ \\
\hline 95 & 7.4328 & 7.4381 & $-0.071 \%$ & $0.043 \%$ \\
\hline 99.5 & 3.6611 & 3.6623 & $-0.034 \%$ & $-11.061 \%$ \\
\hline 99.9 & 3.3172 & 3.3181 & $-0.027 \%$ & $-16.160 \%$ \\
\hline \multicolumn{5}{|c|}{ Weekly Monitoring } \\
\hline 85 & 10.9187 & 10.921 & $-0.021 \%$ & $0.009 \%$ \\
\hline 90 & 10.3118 & 10.3139 & $-0.021 \%$ & $0.088 \%$ \\
\hline 95 & 8.6362 & 8.6381 & $-0.022 \%$ & $-0.970 \%$ \\
\hline 99.5 & 6.1197 & 6.1213 & $-0.027 \%$ & $-14.001 \%$ \\
\hline 99.9 & 5.8716 & 5.8732 & $-0.027 \%$ & $-16.960 \%$ \\
\hline
\end{tabular}

TABLE 3. In-the-money Down and Out Call Option

Parameters: $S=100, K=105, T=0.5$ year, $r=0.05$, $q=0, \sigma=0.25,100$ basis functions.

\begin{tabular}{|c|c|c|c|c|}
\hline \multirow{3}{*}{$\begin{array}{c}\text { Barrier } \\
\text { Level }\end{array}$} & \multirow{3}{*}{$\begin{array}{c}\text { Path Integral } \\
\text { Solution }\end{array}$} & \multirow{3}{*}{$\begin{array}{c}\text { CN } \\
\text { Solution }\end{array}$} & \multicolumn{2}{|c|}{ Percentage Pricing Error } \\
\hline & & & Path & BGK \\
\hline & & & Integral & Correction \\
\hline \multicolumn{5}{|c|}{ Monthly Monitoring } \\
\hline 85 & 5.9226 & 5.9237 & $-0.018 \%$ & $-0.014 \%$ \\
\hline 90 & 5.6068 & 5.6081 & $-0.022 \%$ & $-0.005 \%$ \\
\hline 95 & 4.4945 & 4.4979 & $-0.075 \%$ & $0.058 \%$ \\
\hline 99.5 & 2.3851 & 2.3847 & $0.018 \%$ & $-10.882 \%$ \\
\hline 99.9 & 2.1751 & 2.1751 & $0.001 \%$ & $-15.962 \%$ \\
\hline \multicolumn{5}{|c|}{ Weekly Monitoring } \\
\hline 85 & 5.9545 & 5.9548 & $-0.005 \%$ & $0.000 \%$ \\
\hline 90 & 5.7630 & 5.7642 & $-0.021 \%$ & $0.080 \%$ \\
\hline 95 & 5.0803 & 4.0814 & $-0.022 \%$ & $-0.825 \%$ \\
\hline 99.5 & 3.8346 & 3.8356 & $-0.026 \%$ & $-13.343 \%$ \\
\hline 99.9 & 3.7011 & 3.7021 & $-0.026 \%$ & $-16.258 \%$ \\
\hline
\end{tabular}

TABLE 4. Out-of-the-money Down and Out Call Options

Parameters: $S=100, K=95, T=0.5$ year, $r=0.05$,

$$
q=0, \sigma=0.25,100 \text { basis functions. }
$$

calculated using the path integral method relative to the true value. Following Wei (1998), we also give the percentage errors of prices calculated using the Broadie-Glasserman-Kou (BGK) continuity correction Broadie et al. (1997a). Comparing the percentage errors of the prices derived under the path integral method with those derived under the BGK methods, show that relative errors 
are of comparable magnitude when the barrier level lies away from the current asset price. However, as the barrier level approaches the current asset price, the relative errors producing using the path integral method are much smaller than those using the BGK method. Also, the size of the relative error under the path integral method is relatively stable across barrier levels.

Although not reported in this version of the paper, the computation time required for the path integral method is one of its strengths. For a 1-yeardown-and-out barrier option with weekly monitoring, with 100 basis functions, the algorithm in its current form runs about three times faster than the Crank-Nicholson method to give the same level of accuracy. Of course the computation times for the path integral method increase with the number of basis functions and with the monitoring frequency. However we should point out that the computer codes for both the Hermite expansion method and the Crank-Nicholson method have not been optimised as much as they could be. We leave to future research the task of doing a thorough comparison of the relative efficiency of these and other methods.

\section{Conclusion}

In this paper we have presented a pricing method for the valuation of discretely monitored barrier options in a path integral framework. We show how the backward recursion algorithm of such derivative securities in this framework may be efficiently evaluated by expanding the price in a Fourier-Hermite series as a function of the underlying asset price. The method has the advantage of giving the price as a continuous function of the underlying asset price, hence the hedge ratios can be calculated to a high degree of accuracy with minimal additional computational effort. The method can handle various barrier structures with constant and time varying barrier levels for a variety of option payoffs. The method can be made arbitrarily accurate by increasing the number of basis functionsn in the expansions. Preliminary numerical results show that the method presented is relatively accurate and efficient.

There are several paths for future research. First, it would be of interest to calculate the deltas and delta hedging costs an option in this framework. Second, the approach here could be compound for speed and accuracy with the recent approach of Fusai, Abrahams and Sgarra $(2006)^{5}$ which solves numerically a Wiener-hopf equation. Third, a natural and simple extension of the work presented in this article would be to apply this method to discretely monitored lookback and Parisian options. American versions of these options could also be conveniently handled in this framework.

\footnotetext{
${ }^{5}$ We are grateful to a referee for drawing this paper to our attention.
} 


\section{Appendix}

\section{A The coefficient $a_{0,0}^{k}$}

By definition,

$$
a_{0,0}^{k}=\frac{1}{\sqrt{\pi}} \int_{x_{l}}^{x_{u}} e^{-x^{2}} d x
$$

which by change of variable becomes

$$
\begin{aligned}
a_{0,0}^{k} & =\frac{1}{\sqrt{2 \pi}} \int_{\sqrt{2} x_{l}}^{\sqrt{2} x_{u}} e^{-z^{2} / 2} d z \\
& =\frac{1}{\sqrt{2 \pi}} \int_{-\infty}^{\sqrt{2} x_{u}} e^{-z^{2} / 2} d z-\frac{1}{\sqrt{2 \pi}} \int_{-\infty}^{\sqrt{2} x_{l}} e^{-z^{2} / 2} d z .
\end{aligned}
$$

This can be expressed as

$$
a_{0,0}^{K}=\Phi\left(\sqrt{2} x_{u}\right)-\Phi\left(\sqrt{2} x_{l}\right),
$$

or using the notation as given by equation (15) we have that

$$
a_{0,0}^{k}=P\left(x_{u}, x_{l}\right) .
$$

It is worth noting some some special values for this coefficient

At the pay-off, $k=K$ we have in the case of a call that $x_{l}=0$ and $x_{u} \rightarrow \infty$ so that

$$
a_{0,0}^{k}=P(0, \infty)=\frac{1}{2} .
$$

In the case of a put,we have that $x_{l} \rightarrow-\infty$ and $x_{u}=0$ so that

$$
a_{0,0}^{k}=P(-\infty, 0)=\frac{1}{2} .
$$

Consider the general time step, $k=(K-1, \cdots, 1)$, there are two special cases:

If we have only an upper barrier, then $x_{l} \rightarrow-\infty$ and

$$
a_{0,0}^{k}=P\left(-\infty, x_{u}\right)=\Phi\left(\sqrt{2} x_{u}\right) .
$$

If we have only a lower barrier, then $x_{u} \rightarrow \infty$ and

$$
a_{0,0}^{k}=P\left(x_{l}, \infty\right)=1-\Phi\left(\sqrt{2} x_{l}\right) .
$$




\section{B The coefficient $a_{1,0}^{k}$}

By definition,

$$
a_{1,0}^{k}=\frac{1}{2 v} \frac{1}{\sqrt{\pi}} \int_{x_{l}}^{x_{u}} 2 x e^{-x^{2}} d x
$$

which easily evaluates to

$$
a_{1,0}^{k}=\frac{1}{2 v} \sqrt{2}\left[\frac{1}{\sqrt{2 \pi}} e^{-\left(\sqrt{2} x_{l}\right)^{2} / 2}-\frac{1}{\sqrt{2 \pi}} e^{-\left(\sqrt{2} x_{u}\right)^{2} / 2}\right],
$$

which upon use of the notation $\phi(x)=e^{-x^{2} / 2} / \sqrt{2 \pi}$, becomes

$$
a_{1,0}^{k}=\frac{1}{2 v}\left[\phi\left(\sqrt{2} x_{l}\right)-\phi\left(\sqrt{2} x_{u}\right)\right] .
$$

Using the notation of equation (14) we can also write

$$
a_{1,0}^{k}=\frac{1}{2 v} Q_{0,0}\left(x_{l}, x_{u}\right) .
$$

We note some special values.

At the pay-off, $k=K$ we have in the case of a call that $x_{l}=0$ and $x_{u} \rightarrow \infty$, then

$$
a_{1,0}^{K}=Q_{0,0}(0, \infty)=\frac{1}{2 v \sqrt{\pi}}
$$

In the case of a put, $x_{l} \rightarrow-\infty$ and $x_{u}=0$. then,

$$
a_{1,0}^{k}=Q_{0,0}(-\infty, 0)=\frac{-1}{2 v \sqrt{\pi}} .
$$

Here we see that the difference between the two cases is a simple sign change, that is

$$
a_{1,0}^{k}(\text { Call })=-a_{1,0}^{K}(\text { Put }) .
$$

At the general time step $k$, there are two special cases of interest:If we have only an upper barrier so that $x_{l} \rightarrow-\infty$ then

$$
\begin{aligned}
a_{1,0}^{k} & =\frac{1}{2 v} Q_{0,0}\left(-\infty, x_{u}\right), \\
& =\frac{1}{2 v}\left[R_{0,0}(-\infty)-R_{0,0}\left(x_{u}\right)\right], \\
& =\frac{-\sqrt{2}}{2 v} \phi\left(\sqrt{2} x_{u}\right) .
\end{aligned}
$$

If we have only a lower barrier so that $x_{u} \rightarrow \infty$ then 


$$
\begin{aligned}
a_{1,0}^{k} & =\frac{1}{2 v} Q_{0,0}\left(x_{l}, \infty\right), \\
& =\frac{1}{2 v}\left[R_{0,0}\left(x_{l}\right)-R_{0,0}(\infty)\right], \\
& =\frac{\sqrt{2}}{2 v} \phi\left(\sqrt{2} x_{l}\right) .
\end{aligned}
$$

\section{The coefficient $a_{0,1}^{k}$}

By definition

$$
a_{0,1}^{k}=\frac{1}{\sqrt{\pi}} \int_{x_{l}}^{x_{u}} e^{-x^{2}} 2(v x+\beta) d x,
$$

which can be written

$$
a_{0,1}^{k}=v I_{1}+2 \beta I_{2},
$$

where

$$
I_{1}=\frac{1}{\sqrt{\pi}} \int_{x_{l}}^{x_{u}} 2 x e^{-x^{2}} d x, \quad I_{2}=\frac{1}{\sqrt{\pi}} \int_{x_{l}}^{x_{u}} e^{-x^{2}} d x .
$$

Using results from Appendices A and B, we find that

$$
\begin{aligned}
& I_{1}=\sqrt{2}\left[\phi\left(\sqrt{2} x_{l}\right)-\phi\left(\sqrt{2} x_{u}\right)\right], \\
& I_{2}=\left[\Phi\left(\sqrt{2} x_{u}\right)-\Phi\left(\sqrt{2} x_{l}\right)\right] .
\end{aligned}
$$

Hence on using equation (14) and (15)

$$
a_{0,1}^{k}=v Q_{0,0}\left(x_{l}, x_{u}\right)+2 \beta P\left(x_{u}, x_{l}\right) .
$$

We consider some special values. At the pay-off, $k=K$. In the case of a call we have $x_{l}=0$ and $x_{u} \rightarrow \infty$ so that

$$
a_{0,1}^{k}=v Q_{0,0}(0, \infty)+2 b P(0, \infty)=\frac{1}{2 v \sqrt{\pi}}+\beta .
$$

In the case of a put we have $x_{l} \rightarrow-\infty$ and $x_{u}=0$ so that

$$
a_{0,1}^{K}=v Q_{0,0}(-\infty, 0)+2 \beta P(-\infty, 0)=\frac{-1}{2 v \sqrt{\pi}}+\beta .
$$

At the general time step $k$, there are two special cases of interest.

The case of only an upper barrier $\left(x_{l} \rightarrow-\infty\right)$ so that 


$$
\begin{aligned}
a_{0,1}^{k} & =v Q_{0,0}\left(-\infty, x_{u}\right)+2 \beta P\left(-\infty, x_{u}\right), \\
& =v\left[R_{0,0}(-\infty)-R_{0,0}\left(x_{u}\right)\right]+2 \beta P\left(-\infty, x_{u}\right), \\
& =-v \sqrt{2} \phi\left(\sqrt{2} x_{u}\right)+2 b \Phi\left(\sqrt{2} x_{u}\right) .
\end{aligned}
$$

In the case of only a lower barrier $\left(x_{u} \rightarrow \infty\right)$ we have

$$
\begin{aligned}
a_{0,1}^{k} & =v Q_{0,0}\left(x_{l}, \infty\right)+2 \beta P\left(x_{l}, \infty\right), \\
& =v\left[R_{0,0}\left(x_{l}\right)-R_{0,0}(\infty)\right]+2 \beta P\left(x_{l}, \infty\right), \\
& =v \sqrt{2} \phi\left(\sqrt{2} x_{l}\right)+2 \beta\left[1-\Phi\left(\sqrt{2} x_{l}\right)\right] .
\end{aligned}
$$

\section{The coefficient $a_{1,1}^{k}$}

By definition

$$
a_{1,1}^{k}=\frac{1}{2 v \sqrt{\pi}} \int_{x_{l}}^{x_{u}} e^{-x^{2}}(2 x) 2(v x+\beta) d x,
$$

which when integrating by parts and using (14) and (15) can be written as

$$
a_{1,1}^{k}=\frac{1}{2 v} Q_{0,1}\left(x_{l}, x_{u}\right)+P\left(x_{l}, x_{u}\right) .
$$

At the pay-off, $k=K$, we have two special cases. The call for which $x_{l}=0$ and $x_{u} \rightarrow \infty$ so that

$$
\begin{aligned}
a_{1,1}^{K} & =\frac{1}{2 v} Q_{0,1}(0, \infty)+P(0, \infty), \\
& =\frac{\beta}{2 v \sqrt{\pi}}+\frac{1}{2},
\end{aligned}
$$

and the put for which $x_{l} \rightarrow-\infty$ and $x_{u}=0$ so that

$$
\begin{aligned}
a_{1,1}^{K} & =\frac{1}{2 v} Q_{0,1}(-\infty, 0)+P(-\infty, 0), \\
& =\frac{-\beta}{v \sqrt{\pi}}+\frac{1}{2} .
\end{aligned}
$$

At the general time step $k$, there are two special cases of interest. Only upper barrier $\left(x_{l} \rightarrow-\infty\right)$ so that 


$$
\begin{aligned}
a_{1,1}^{k} & =\frac{1}{2 v} Q_{0,1}\left(-\infty, x_{u}\right)+P\left(-\infty, x_{u}\right), \\
& =\frac{1}{2 v}\left[R_{0,1}(-\infty)-R_{0,1}\left(x_{u}\right)\right]+P\left(-\infty, x_{u}\right), \\
& =\frac{-\sqrt{2}}{2 v} H_{1}\left(v x_{u}+b\right)+\Phi\left(\sqrt{2} x_{u}\right) .
\end{aligned}
$$

The case of only lower barrier $\left(x_{u} \rightarrow \infty\right)$ so that

$$
\begin{aligned}
a_{1,1}^{k} & =\frac{1}{2 v} Q_{0,1}\left(x_{l}, \infty\right)+P\left(x_{l}, \infty\right), \\
& =\frac{1}{2 v}\left[R_{0,1}\left(x_{l}\right)-R_{0,1}(\infty)\right]+P\left(x_{l}, \infty\right), \\
& =\frac{\sqrt{2}}{2 v} H_{1}\left(v x_{l}+\beta\right)+\left[1-\Phi\left(\sqrt{2} x_{l}\right)\right] .
\end{aligned}
$$

\section{E The coefficient $a_{m, o}^{k}$}

By definition

$$
a_{m, o}^{k}=\frac{1}{2^{m} m ! v^{m}} \frac{1}{\sqrt{\pi}} \int_{x_{l}}^{x_{u}} e^{-x^{2}} H_{m}(x) d x .
$$

Using the three-term reccurence relation,

$$
H_{m}(x)=2 x H_{m-1}(x)-2(m-1) H_{m-2}(x)
$$

equation (80) can be written as

$$
a_{m, 0}^{k}=W-\frac{1}{2 v^{2}} a_{m-2, o}^{k},
$$

where we set

$$
W=\frac{1}{2^{m} m ! v^{m}} \frac{1}{\sqrt{\pi}} \int_{x_{l}}^{x_{u}} 2 x e^{-x^{2}} H_{m-1}(x) d x .
$$

Integrating by parts we find that

$$
W=\frac{\sqrt{2}}{2^{m} m ! v^{m}}\left[H_{m-1}\left(x_{l}\right) \phi\left(\sqrt{2} x_{l}\right)-H_{m-1}\left(x_{u}\right) \phi\left(\sqrt{2} x_{u}\right)\right]+\frac{1}{2 m v^{2}} a_{m, o}^{k},
$$

which when substituted back into (82) gives

$$
a_{m, o}^{k}=\frac{\sqrt{2}}{2^{m} m ! v^{m}}\left[H_{m-1}\left(x_{l}\right) \phi\left(\sqrt{2} x_{l}\right)-H_{m-1}\left(x_{u}\right) \phi\left(\sqrt{2} x_{u}\right)\right] .
$$

Use of (14) finally allows us to write 


$$
a_{m, 0}^{k}=\frac{1}{2 m v} Q_{m-1, o}\left(x_{l}, x_{u}\right) .
$$

At the pay-off, $k=K$, we have two special cases. The call for which $x_{l}=0$ and $x_{u} \rightarrow \infty$ so that

$$
\begin{aligned}
a_{m, o}^{K} & =\frac{1}{2 m v} Q_{m-1, o}(0, \infty), \\
& =\frac{1}{2 m v}\left[R_{m-1,0}(0)-R_{m-1,0}(0)\right], \\
& \left.=\frac{1}{2^{m} m ! v^{m}} \frac{H_{m-1}(0)}{\sqrt{\pi}}, \quad \quad \quad \text { (use of }(13)\right), \\
& =\frac{1}{2 m v \sqrt{\pi}} L_{m-1}(0), \quad(\text { use of }(11)) .
\end{aligned}
$$

The put for which $x_{l} \rightarrow-\infty$ and $x_{u}=0$ so that

$$
\begin{aligned}
a_{m, o}^{K} & =\frac{1}{2 m v} Q_{m-1, o}(-\infty, 0), \\
& =\frac{1}{2 m v}\left[R_{m-1,0}(-\infty)-R_{m-1,0}(0)\right], \\
& =\frac{-1}{2^{m} m ! v^{m}} \frac{H_{m-1}(0)}{\sqrt{\pi}}, \quad(\text { use of }(13)), \\
& =\frac{-1}{2 m v \sqrt{\pi}} L_{m-1}(0), \quad(\text { use of }(11)) .
\end{aligned}
$$

At the general time step $k$, there are two special cases of interest. Only upper barrier $\left(x_{l} \rightarrow-\infty\right)$ when,

$$
\begin{aligned}
a_{m, o}^{k} & =\frac{1}{2 m v} Q_{m-1, o}\left(-\infty, x_{u}\right), \\
& =\frac{1}{2 m v}\left[R_{m-1, o}(-\infty)-R_{m-1, o}\left(x_{u}\right)\right], \\
& \left.=\frac{-\sqrt{2}}{2^{m} m ! v^{m}} H_{m-1}\left(x_{u}\right) \phi\left(\sqrt{2} x_{u}\right), \quad \text { (use of }(13)\right) \\
& \left.=\frac{-\sqrt{2}}{2 m v} L_{m-1}\left(x_{u}\right) \phi\left(\sqrt{2} x_{u}\right), \quad \text { (use of }(11)\right) .
\end{aligned}
$$

Only lower barrier $\left(x_{u} \rightarrow \infty\right)$ when 
so finally

$$
\begin{aligned}
a_{m, o}^{k} & =\frac{1}{2 m v} Q_{m-1, o}\left(x_{l}, \infty\right) \\
& =\frac{1}{2 m v}\left[R_{m-1, o}\left(x_{l}\right)-R_{m-1, o}(\infty)\right] \\
& =\frac{\sqrt{2}}{2^{m} m ! v^{m}} H_{m-1}\left(x_{l}\right) \phi\left(\sqrt{2} x_{l}\right), \quad(\text { use of }(13))
\end{aligned}
$$

$$
\left.=\frac{\sqrt{2}}{2 m v} L_{m-1}\left(x_{l}\right) \phi\left(\sqrt{2} x_{l}\right), \quad \text { (use of }(11)\right) .
$$

\section{F The coefficient $a_{0, n}^{k}$}

By definition

$$
a_{o, n}^{k}=\frac{1}{\sqrt{\pi}} \int_{x_{l}}^{x_{u}} e^{-x^{2}} H_{n}(v x+b) d x .
$$

Using the three-term reccurance relation,

$$
H_{n}(v x+b)=2(v x+\beta) H_{n-1}(v x+b)-2(n-1) H_{n-2}(v x+b),
$$

it follows that

$$
a_{0, n}^{k}=v W+2 \beta a_{o, n-1}^{k}-2(n-1) a_{o, n-2}^{k},
$$

where we set

$$
W=\frac{1}{\sqrt{\pi}} \int_{x_{l}}^{x_{u}} 2 x e^{-x^{2}} H_{n-1}(v x+\beta) d x .
$$

Integrating by parts, we find that

$$
W=\left[-H_{n-1}(v x+\beta) \frac{e^{-x^{2}}}{\sqrt{\pi}}\right]_{x_{l}}^{x_{u}}+2 v(n-1) a_{0, n-2}^{k},
$$

which reduces (93) to

$$
\begin{aligned}
a_{o, n}^{k} & =v \sqrt{2}\left[H_{n-1}\left(v x_{l}+\beta\right) \phi\left(\sqrt{2} x_{l}\right)-H_{n-1}\left(v x_{u}+\beta\right) \phi\left(\sqrt{2} x_{u}\right)\right] \\
& +2 \beta a_{o, n-1}^{k}+2\left(v^{2}-1\right)(n-1) a_{o, n-2}^{k}
\end{aligned}
$$

which by use of (14) can be written as

$$
a_{o, n}^{k}=v Q_{o, n-1}\left(x_{l}, x_{u}\right)+2 \beta a_{o, n-1}^{k}+2\left(v^{2}-1\right)(n-1) a_{o, n-2}^{k} .
$$

At the pay-off, $k=K$, we have two special cases. The call for which $x_{l}=0$ and $x_{u} \rightarrow \infty$, so that 


$$
\begin{aligned}
a_{o, n}^{K} & =v Q_{o, n-1}(0, \infty)+2 \beta a_{o, n-1}^{K}+2\left(v^{2}-1\right)(n-1) a_{o, n-2}^{K} \\
& =v\left[R_{o, n-1}(0)-R_{o, n-1}(\infty)\right]+2 \beta a_{o, n-1}^{K}+2\left(v^{2}-1\right)(n-1) a_{o, n-2}^{K},
\end{aligned}
$$

which by use of (14) finally reduces to

$$
a_{o, n}^{K}=\frac{v}{\sqrt{\pi}} H_{n-1}(\beta)+2 \beta a_{o, n-1}^{K}+2\left(v^{2}-1\right)(n-1) a_{o, n-2}^{K} .
$$

The put for which $x_{l} \rightarrow-\infty$ and $x_{u}=0$ so that

$$
\begin{aligned}
a_{o, n}^{k} & =v Q_{o, n-1}(-\infty, 0)+2 \beta a_{o, n-2}^{K}+2\left(v^{2}-1\right)(n-1) a_{o, n-2}^{K}, \\
& =v\left[R_{o, n-1}(-\infty)-R_{o, n-1}(0)\right]+2 b a_{o, n-1}^{K}+2\left(v^{2}-1\right)(n-1) a_{o, n-2}^{K},
\end{aligned}
$$

which by use of (14)finally reduces to

$$
a_{o, n}^{K}=\frac{-v}{\sqrt{\pi}} H_{n-1}(b)+2 \beta a_{o, n-1}^{K}+2\left(v^{2}-1\right)(n-1) a_{o, n-2}^{K} .
$$

At the general time step $k$, there are two special cases of interest. The case of only upper barrier $\left(x_{l} \rightarrow-\infty\right)$, when

$$
\begin{aligned}
a_{o, n}^{k} & =v Q_{o, n-1}\left(-\infty, x_{u}\right)+2 \beta a_{o, n-1}^{k}+2\left(v^{2}-1\right)(n-1) a_{o, n-2}^{k}, \\
& =v\left[R_{o, n-1}(-\infty)-R_{o, n-1}\left(x_{u}\right)\right]+2 b a_{o, n-1}^{k}+2\left(v^{2}-1\right)(n-1) a_{o, n-2}^{k},
\end{aligned}
$$

which by use of (14) reduces to

$$
a_{o, n}^{k}=-v \sqrt{2} H_{n-1}\left(v x_{u}+\beta\right) \phi\left(\sqrt{2} x_{u}\right)+2 \beta a_{o, n-1}^{k}+2\left(v^{2}-1\right)(n-1) a_{o, n-2}^{k} .
$$

The case of only lower barrier $\left(x_{u} \rightarrow \infty\right)$, when

$$
\begin{aligned}
a_{o, n}^{k} & =v Q_{o, n-1}\left(x_{l}, \infty\right)+2 \beta a_{o, n-1}^{k}+2\left(v^{2}-1\right)(n-1) a_{o, n-2}^{k}, \\
& =v\left[R_{o, n-1}\left(x_{l}\right)-R_{o, n-1}(\infty)\right]+2 \beta a_{o, n-1}^{k}+2\left(v^{2}-1\right)(n-1) a_{o, n-2}^{k},
\end{aligned}
$$

which by use of (14) reduces to

$$
a_{o, n}^{k}=v \sqrt{2} H_{n-1}\left(v x_{l}+\beta\right) \phi\left(\sqrt{2} x_{l}\right)+2 \beta a_{o, n-1}^{k}+2\left(v^{2}-1\right)(n-1) a_{o, n-2}^{k} .
$$




\section{G The coefficient $a_{m, n}^{k}$}

By definition

$$
a_{m, n}^{k}=\frac{1}{2^{m} m ! v^{m}} \frac{1}{\sqrt{\pi}} \int_{x_{l}}^{x_{u}} e^{-x^{2}} H_{m}(x) H_{n}(v x+b) d x .
$$

Using the three-term reccurence relation,

$$
H_{m}(x)=2 x H_{m-1}(x)-2(m-1) H_{m-2}(x),
$$

equation (106) can be written as

$$
a_{m, n}^{k}=W-\frac{1}{2 m v^{2}} a_{m-2, n}^{k}
$$

where we set

$$
W=\frac{1}{2^{m} m ! v^{m}} \frac{1}{\sqrt{\pi}} \int_{x_{l}}^{x_{u}} 2 x e^{-x^{2}} H_{m-1}(x) H_{n}(v x+b) d x .
$$

Integrating by parts we find that

$$
\begin{aligned}
W & =\frac{\sqrt{2}}{2^{m} m ! v^{m}}\left[H_{m-1}\left(x_{l}\right) H_{n}\left(v x_{l}+\beta\right) \phi\left(\sqrt{2} x_{l}\right)-H_{m-1}\left(x_{u}\right) H_{n}(v x+\beta) \phi\left(\sqrt{2} x_{u}\right)\right] \\
& +\frac{1}{2 m v^{2}} a_{m-2, n}^{k}+\frac{n}{m} a_{m-1, n-1}^{k},
\end{aligned}
$$

which by use of (15) can be written as

$$
W=\frac{1}{2 m v} Q_{m-1, n}+\frac{1}{2 m v^{2}} a_{m-2, n}^{k}+\frac{n}{m} a_{m-1, n-1}^{k} .
$$

Finally, substituting (111) back into (108) yields

$$
a_{m, n}^{k}=\frac{1}{2 m v} Q_{m-1, n}+\frac{n}{m} a_{m-1, n-1}^{k} .
$$

At the pay-off, $k=K$, we have two special cases. The call for which $x_{l}=0$ and $x_{u} \rightarrow \infty$, so that

$$
\begin{aligned}
a_{m, n}^{K} & =\frac{1}{2 m v} Q_{m-1, n}(0, \infty)+\frac{n}{m} a_{m-1, n-1}^{K}, \\
& \left.=\frac{1}{2 m v}\left[R_{m-1, n}(0)-R_{m-1, n}(\infty)\right] \quad \text { (use of }(14)\right) \\
& +\frac{n}{m} a_{m-1, n-1}^{k},
\end{aligned}
$$

which by use of (13) reduces to 


$$
a_{m, n}^{K}=\frac{\sqrt{2}}{2 m v \sqrt{\pi}} L_{m-1}(0) H_{m}(\beta)+\frac{n}{m} a_{m-1, n-1}^{K} .
$$

The put for which $x_{l} \rightarrow-\infty$ and $x_{u}=0$, so that

$$
\begin{aligned}
a_{m, n}^{K} & =\frac{1}{2 m v} Q_{m-1, n}(-\infty, 0)+\frac{n}{m} a_{m-1, n-1}^{K}, \\
& =\frac{1}{2 m v}\left[R_{m-1, n}(-\infty)-R_{m-1, n}(0)\right]+\frac{n}{m} a_{m-1, n-1}^{K},
\end{aligned}
$$

which by use of (13) reduces to

$$
a_{m, n}^{K}=\frac{-\sqrt{2}}{2 m v \sqrt{\pi}} L_{m-1}(0) H_{m}(\beta)+\frac{n}{m} a_{m-1, n-1}^{K} .
$$

At the general time step $k$, there are two special cases. The case of only upper barrier $\left(x_{l} \rightarrow-\infty\right)$, when

$$
\begin{aligned}
a_{m, n}^{k} & =\frac{1}{2 m v} Q_{m-1, n}\left(-\infty, x_{u}\right)+\frac{n}{m} a_{m-1, n-1}^{k} \\
& =\frac{1}{2 m v}\left[R_{m-1, n}(-\infty)-R_{m-1, n}\left(x_{u}\right)\right]+\frac{n}{m} a_{m-1, n-1}^{k}
\end{aligned}
$$

which by use of (13) finally reduces to

$$
a_{m, n}^{k}=\frac{-\sqrt{2}}{2 m v} L_{m}\left(x_{u}\right) H_{n}\left(v x_{u}+\beta\right) \phi\left(\sqrt{2} x_{u}\right)+\frac{n}{m} a_{m-1, n-1}^{k} .
$$

The case of only lower barrier $\left(x_{u} \rightarrow \infty\right)$, when

$$
\begin{aligned}
a_{m, n}^{k} & =\frac{1}{2 m v} Q_{m-1, n}\left(x_{l}, \infty\right)+\frac{n}{m} a_{m-1, n-1}^{k} \\
& =\frac{1}{2 m v}\left[R_{m-1, n}\left(x_{l}\right)-R_{m-1, n}(\infty)\right]+\frac{n}{m} a_{m-1, n-1}^{k},
\end{aligned}
$$

which by use of (13) finally reduces to

$$
a_{m, n}^{k}=\frac{\sqrt{2}}{2 m v} L_{m-1}(x) H_{n}\left(v x_{l}+b\right)+\frac{n}{m} a_{m-1, n-1}^{k} .
$$

\section{H Useful Notation}

$$
\begin{gathered}
P\left(x_{l}, x_{u}\right)=\Phi\left(\sqrt{2} x_{u}\right)=\Phi\left(\sqrt{2} x_{l}\right) \\
Q_{m, n}\left(x_{l}, x_{u}\right)=R_{m, n}\left(x_{l}\right)-R_{m, n} x_{u}
\end{gathered}
$$


where

$$
R_{m, n}(x)=\sqrt{2} L_{m}(x) H_{n}(v x+b) \phi(\sqrt{2} x)
$$

with

$$
L_{m}(x)=\frac{1}{2^{m} m ! V^{m}} H_{m}(x)
$$

and

$$
\phi(x)=\frac{1}{\sqrt{2 \pi}} e^{\frac{-x^{2}}{2}}, \quad \Phi(x)=\frac{1}{\sqrt{2 \pi}} \int_{0}^{x} e^{\frac{-\xi^{2}}{2}} d \xi
$$

furthermore,

$$
H_{n}(x)=2 x H_{n-1}(x)-2(n-1) H_{n-2}(x)
$$

with

$$
\begin{gathered}
H_{o}(x)=1 \quad \text { and } \quad H_{1}(x)=2 x \\
L_{m}(x)=\frac{x}{m V} L_{m-1}(x)-\frac{1}{2 m V^{2}} L_{m-2}(x)
\end{gathered}
$$

with

$$
L_{o}(x)=1 \quad \text { and } \quad L_{1}(x)=\frac{x}{V}
$$

Let us define

$$
L_{m}(x)=\frac{1}{2^{m} m ! V^{m}} H_{m}(x)
$$

Using the three term reccurance relation

$$
H_{m}(x)=2 x H_{m-1}(x)-2(m-1) H_{m-2}(x)
$$

and nothing that

$$
\begin{gathered}
\frac{2}{2^{m} m ! V^{m}}=\frac{1}{m V}\left[\frac{1}{2^{m-1}(m-1) ! V^{m-1}}\right] \\
\frac{2(m-1)}{2^{m} m ! V^{m}}=\frac{1}{2 m V^{2}}\left[\frac{1}{2^{m-2}(m-2) ! V^{m-2}}\right]
\end{gathered}
$$

we instantly have that

$$
L_{m}(x)=\frac{x}{m V} L_{m-1}(x)-\frac{1}{2 m V^{2}} L_{m-2}(x)
$$

with

$$
\begin{aligned}
& L_{o}(x)=1 \\
& L_{1}(x)=\frac{x}{v}
\end{aligned}
$$

thus the $L_{m}$ 's can be generated easily using (135), (136), (137).

Now, let us define 


$$
R_{m, n}(x)=\sqrt{2} L_{m}(x) H_{n}(v x+b) \phi(\sqrt{2} x)
$$

Special values $(x=0)$

$$
R_{m, n}(0)=\frac{1}{\sqrt{\pi}} L_{m}(0) H_{n}(b)
$$

and in particular,

$$
\begin{aligned}
R_{m, 0}(0) & =\frac{1}{\sqrt{\pi}} L_{m}(0) \\
R_{0, n}(0) & =\frac{1}{\sqrt{\pi}} H_{n}(b)
\end{aligned}
$$

Special values $(x \rightarrow \infty, x \rightarrow-\infty)$

$$
\begin{aligned}
& \lim _{x \rightarrow-\infty} R_{m, n}(x)=0 \\
& \lim _{x \rightarrow \infty} R_{m, n}(x)=0
\end{aligned}
$$

Furthermore, using the above notation,

$$
Q_{m, n}\left(x_{l}, x_{u}\right)=R_{m, n}\left(x_{l}\right)-R_{m, n}\left(x_{u}\right)
$$

In general,

$$
P\left(x_{l}, x_{u}\right)=\left[\Phi\left(\sqrt{2} x_{u}\right)-\Phi\left(\sqrt{2} x_{l}\right)\right]
$$

Special cases:

$$
\begin{aligned}
P(-\infty, \infty) & =1 \\
P(-\infty, 0) & =\frac{1}{2} \\
P(0, \infty) & =\frac{1}{2} \\
P\left(-\infty, x_{u}\right) & =\Phi\left(\sqrt{2} x_{u}\right) \\
P\left(x_{l}, \infty\right) & =1-\Phi\left(\sqrt{2} x_{l}\right) \\
P\left(x_{l}, 0\right) & =\frac{1}{2}-\Phi\left(\sqrt{2} x_{l}\right) \\
P\left(0, x_{u}\right) & =\Phi\left(\sqrt{2} x_{u}\right)-\frac{1}{2}
\end{aligned}
$$




\section{References}

Boyle, P. and Tian, Y.: 1997, An Explicit Finite Difference Approach to the Pricing of Barrier Options, Working Paper, Universit of Waterloo and University of Cinicinnati .

Broadie, M., Glasserman, P. and Kou, S.: 1997a, A continuity Correction for Discrete Barrier Options, Mathematical Finance 7(4), 328-349.

Broadie, M., Glasserman, P. and Kou, S.: 1997b, Connecting Discrete and Continuous Path-Dependent Options, Working Paper, Columbia Business School and University of Michigan .

Chance, D.: 1994, The pricing and Hedging of Limited Exercise Caps and Spreads, Journal of Financial Res. 17, 561-584.

Chiarella, C. and El-Hassan, N.: 1997, Evaluation of Derivative Security Prices in the Heath-Jarrow-Morton Framework as Path Integrals Using Fast Fourier Transform Techniques, The Journal of Financial Engineering 6(2), 121-147.

Chiarella, C., El-Hassan, N. and Kucera, A.: 1999, Evaluation Of American Option Prices In a Path Integral Framework Using Fourier-Hermite Series Expansions, Journal of Economic and Dynamic Control 23, 1387-1424.

Dash, J.: 1988, Path Integrals and Options - Part I, CRNS Preprints .

Eydeland, A.: 1994, A Fast Algorithm for Computing Integrals in Function Spaces: Financial Applications, Computational Economics 7(4), 277-285.

Figlewski, S. and Gao, B.: 1997, The Adaptive Mesh Model: A New Approach to Efficient Option Pricing, Working Paper, Salomon Centre, NYU S-97-5 .

Fusai, G., Abrahams, I. and Sgarra, C.: 2006, An exanct analytical solution for discrete barrier options, Finance and Stochastic 10, 1-26.

Heynen, R. and Kat, H.: 1996, Discrete Partial Barrier Options with a Moving Barrier, Journal of Financial Engineering 5(3), 199-209.

Kat, H.: 1995, Pricing Lookback Options Using Binomail Trees: An Evaluation, Journal of Financial Engineering 4, 375-397.

Levy, E. and Mantion, F.: 1997, Discrete By Nature, Risk 10(1), 8-22.

Linetsky, V.: 1997, The Path Integral Approach to Financial Modeling and Option Pricing, Computational Economics 11(1), 129-163.

Makici, M.: 1995, Path Integral Monte Carlo Method for Valuation of Derivative Securities: Algorithm and Parallel Implementation, NPAC Technical Report SCCS 650 .

Merton, R.: 1973, The Theory of Rational Option Pricing, Bell Journal of Economics and Management Science 4, 141-183.

Rubinstein, M. and Reiner, E.: 1991, Breaking Down the Barriers, Risk 4(4), 28-35.

Sullivan, M. A.: 2000, Pricing Discretely Monitored Barrier Options, Journal of Computational Finance 3(4), 35-54.

Tavella, D.: 2002, Quantitative Methods in Derivatives Pricing, Wiley Finance .

Tian, Y.: 1996, Breaking the Barrier with a Two-Stage Binomial Model, Working Paper, University of Cincicinnati .

Wei, J.: 1998, Valuation of Discrete Barrier Options by Interpolations, Journal of Derivatives 6(1), 51-73. 